\title{
Arguments en faveur de la double origine des Nématodes néarctiques du genre Heligmosomoides Hall, 1916
}

\author{
par Marie-Claude DURETTE-DESSET, John M. KINSELLA \\ et Donald J. FORRESTER
}

Laboratoire de Zoologie (Vers) associé au C.N.R.S. ( $\mathrm{P}^{\mathbf{r}}$ A.-G. ChaBaud), Muséum national d'Histoire naturelle, 57, rue Cuvier, F 75005 Paris

et Department of Veterinary Science, University of Florida, Gainesville, Florida (U.S.A.)

\section{Résumé}

1) Description d'une nouvelle espèce: Heligmosomoides douglasi parasite d'un Cricétidé Reithrodontomys raviventris, originaire de Californie (U.S.A.). Cette espèce est caractérisée par des spicules courts et un nombre élevé de crêtes cuticulaires. Ses caractères morphologiques la rapprochent des autres Heligmosomoides néarctiques. Le seul point particulier est sa présence chez un Cricétidé et non un Miorotidé.

2) Contrairement à l'opinion généralement admise Heligmosomoides polygyrus polygyrus (Dujardin, 1845) (= Nematospiroides dubius Baylis, 1926), parasite d'Apodemus sylvaticus en Europe occidentale, n'a pas été retrouvé chez les Rongeurs d'Amérique du Nord. Ce sont 2 sous-espèces du polygyrus morphologiquement distinguables, que l'on rencontre chez les Rongeurs de la région néarctique :

a) $H$. polygyrus bakeri n. sub.sp., parasite de Mus musculus (Muridés) et de Reithrodontomys megalotis et Reithrodontomys raviventris (Cricétidés), est caractérisé par une augmentation relative du nombre des crêtes cuticulaires par rapport à la longueur du corps, comme chez H. p. corsicus. Mais il se distingue du précédent par 2 caractères: la bourse caudale est plus développée et la côte 4 gauche est presqu'aussi longue que la côte 5 .

b) $H$. polygyrus americanus n. sub. sp., parasite de Phenacomys intermedius (Miorotidé) est oaractérisé par une diminution 
relative du nombre des crêtes cuticulaires par rapport à la longueur du corps. En outre les côtes 8 présentent une branche interne à leur extrémité proximale et les spicules sont plus longs.

La présence de ces 2 sous-espèces peut s'expliquer, nous semble-t-il, par un double phénomène de capture: Le polygyrus européen aurait été introduit en Amérique du Nord par les Souris grises Mus musculus et aurait subi une légère sub-spéciation pour donner H. p. bakeri. A partir de cette forme, la sous-espèce du Phenacomys se différencie avec une forte sub-spéciation (allongement des spicules, diminution des crêtes cuticulaires).

Dans notre conception, l'introduction des Heligmosomoides en Amérique du Nord s'est donc produite de 2 façons:

a) L'introduction d'un premier groupe, essentiellement inféodé aux Microtidés, a eu lieu au Pleistocène et s'est effectuée par le détroit de Bering corrélativement au passage des hôtes.

b) L'introduction d'un second groupe, connu actuellement par 2 sous-espèces du polygyrus européen, a dû se produire beaucoup plus récemment lorsque Mus musculus est arrivée en Amérique du Nord par passage sur bateau à travers l'Atlantique (fig. 5).

3) Heligmosomoides montanus Durette-Desset 1967, parasite primitivement décrit chez Microtus longicaudus d'Alaska, a été trouvé pour la première fois chez Microtus californicus en Californie.

\section{Summary}

Arguments for the twofold origin of nearctic Nematodes of the genus Heligmosomoides Hall, 1916.

1) Heligmosomoides douglasi n. sp., a parasite of a Cricetid, Reithrodontomys raviventris, in California (U.S.A.) is described. This species is characterized by short spicules and a large number of cuticular ridges. Its morphological characters resemble those of other nearotic Heligmosomoides. The only unusual point is its presence in a Cricetid and not a Microtine.

2) Contrary to general opinion, Heligmosomoides polygyrus polygyrus (Dujardin, 1845) (= Nematospiroides dubius Baylis, 1926), a parasite of Apodemus sylvaticus in western Europe has not been found in North American rodents. We have found two subspecies of polygyrus, morphologically distinguishable, in rodents from the nearctic region:

a) $H$. polygyrus bakeri n. sub. sp., a parasite of Mus musculus (Muridae), Reithrodontomys megalotis, and Reithrodontomys raviventris (Cricetidae), is characterized by a relative increase in the number of cuticular ridges with respect to body length, as in H.p. corsicus. It is distinguished, however, by two characters: the 
bursa is larger and the externolateral ray is almost as long as the mediolateral.

b) $H$. polygyrus americanus $\mathrm{n}$. sub. sp., a parasite of Phenacomys intermedius (Microtidae), is characterized by a relative decrease in the number of cuticular ridges with respect to the body length. In addition, the externodorsal rays have an internal branch at their proximal extremity and the spicules are longer.

We feel that the presence of these two subspecies can be explained by the concept of a double \&phenomenon of capture »: the European polygyrus has been introduced into North America by the house mouse, Mus musculus and has undergone a weak subspeciation to become H.p.bakeri. The subspecies in Phenacomys was derived from th:s form and has undergone a strong subspeciation (elongation of spicules, decrease in the number of cuticular ridges).

The introduction of Heligmosomoides into North America has probably occurred in two ways:

a) The introduction of a first group, infecting primarily Microtine rodents, occurred in the Pleistocene, when migrating hosts crossed the Bering land bridge.

b) The introduction of a second group, consisting of two subspecies of the European polygyrus, occurred much more recently when Mus musculus was brought to North America in ships crossing the Atlantic (fig. 5).

3) Heligmosomoides montanus Durette-Desset, 1967, a parasite originally described from Microtus longicaudus in Alaska, has been found for the first time in Microtus californicus in California.

\section{INTRODUCTION}

Les Heligmosomoides de Rongeurs néarctiques ont un statut taxonomique qui n'est pas définitivement élucidé. Un grand nombre de travaux expérimentaux portent sur un parasite isolé soit de Mus musculus soit de Peromyscus maniculatus et qui est nommé «Nematospiroides dubius».

Durette-Desset a montré (1968 a) que ce taxon est synonyme d'Heligmosomoides polygyrus (Dujardin, 1845) qui est, en réalité, un parasite d'Apodemus sylvaticus en France (1). Par ailleurs, Forrester (1971) s'est inquiété récemment :

1) du fait qu'une souche soi-disant isolée de Peromyscus ne puisse plus infester ce Rongeur,

(1) Dans ce travail (1968 a), l'espèce polygyrus est citée sous le binôme Heligmosomoides polygyrus, p. 387 et sous celui d'Heligmosomum polygyrum, p. 393. Le résumé anglais a été écrit postérieurement à l'article principal au moment où l'auteur rétablissait le genre Heligmosomoides Hail, 1916. En fait, le taxon correct de cette espèce doit s'écrire Heligmosomoides polygyrus (Dujardin, 1845). 
2) du fait que le matériel américain et le matériel européen paraissent montrer de légères différences.

Il nous a donc semblé utile par une étude comparée générale de revoir le statut de «Nematospiroides dubius » nord-américain en particulier et des autres Heligmosomoides trouvés dans cette région.

\section{DONNEES BIBLIOGRAPHIQUES}

Roé (1929) décrit quelques Nématodes d'une «Souris sauvage» de New Jersey sous le nom de Sincosta aberrans. Dikmans (1940) examine le matériel de Roé ainsi que des Nématodes récoltés par G. M. Spurlock chez des Mus musculus originaires de Conway Ranch, Woodland, California (voir Spurlock, 1943). Il identifie le matériel de Roé et celui de Spurlock à Nematospiroides dubius Baylis, 1926. Ehrenford (1954) établit une souche de «Nematospiroides dubius » chez la Souris blanche à l'University of California, Davis, California. L'origine de cette souche était Peromyscus maniculatus, également originaire de Conway Ranch, Woodland, California. Cette souche (souche 50 de Forrester, 1971) a été passée successivement au laboratoire chez des Souris blanches pendant 21 ans et a été à la base de beaucoup de recherches expérimentales (cf. Baker, 1954 ; Cross, 1960 ; Douglas et coll., 1956). Dans ces travaux et dans de nombreux autres, l'espèce a été désignée sous le nom de Nematospiroides dubius Baylis, 1926.

\section{DONNEES MORPHOLOGIQUES NOUVELLES}

Grâce à un abondant matériel récolté en Amérique du Nord par deux d'entre nous, il a été possible de mettre en évidence plusieurs faits :

1) L'espèce-type Heligmosomoides polygyrus polygyrus (Dujardin, 1845) n'a jamais été retrouvée dans le matériel étudié.

2) Ce qui a été décrit jusqu'à présent comme «Nematospiroides dubius 》 dans la région néarctique correspond en fait à deux sous-espèces :

a) Heligmosomoides polygyrus bakeri n. subsp. parasite les Muridés (Mus musculus), les Cricétidés du genre Reithrodontomys et, peut-être, mais de façon très rare, Peromyscus maniculatus. Chez cet hôte, la présence du parasite reste très douteuse. Le fait que la souche d'Ehrenford (souche 50) n'infeste pas le Peromyscus (cf. Forrester, 1971) signifie, à notre avis, qu'il y a eu confusion avec le parasite de Mus, soit au départ, soit au cours des passages successifs chez les Souris de laboratoire.

b) Heligmosomoides polygyrus americanus n. subsp. parasite un Microtidé: Phenacomys intermedius.

3) Par ailleurs, en dehors du groupe polygyrus, nous avons trouvé chez un Cricétidé (Reithrodontomys) une nouvelle espèce Heligmosomoides douglasi $\mathrm{n}$. sp. qui est à rapprocher des autres Heligmosomoides parasites de Microtidés néarctiques.

4) Enfin, nous avons trouvé chez Microtus californicus, l'espèce Heligmosomoides montanus Durette-Desset, 1967, décrit chez $M$. longicaudus d'Alaska. 


\section{DESCRIPTION DES ESPECES}

\section{Heligmosomoides polygyrus bakeri n. sub.sp.}

Matériel étudié : très nombreux ô et + ,

origine géographique : California U.S.A.

collection D. J. Forrester.

1) Chez Mus musculus (Muridés).

Matériel type: tube $685 \mathrm{M}$ (DJF 37).

Origine : $\subsetneq$ de Mus musculus, piégée le 15 novembre 1966 à Conway Ranch, Woodland.

Autre matériel: tubes $655 \mathrm{M}$ à $683 \mathrm{M}$ (DJF 1 à 29). Origine: Mus musculus piégés du 16 au 26 décembre 1966, Conway, Ranch, Wooddland (1) ; tubes 684 M - $686 \mathrm{M}$ (DJF 36 -38). Origine : 2 Mus musculus ơ piégés le 19 novembre 1966 à Conway Ranch, Woodland; tube 687 M (DJF 39). Origine: Mus musculus + piégée le 23 juin 1966 et tuée le 30 août 1966, Davis ; tube 689 M (DJF 35). Origine: Mus musculus ơ piégé le 11 mars 1967 à Novato Creek, Black Point.

Souches de laboratoire.

Souche 67-tube $688 \mathrm{M}$ (DJF 34). Matériel récolté à partir de Souris sauvages et pures Mus musculus et de quelques Reithrodontomys raviventris infestés (Expérience 67-8, 5 mai 1967) par des larves obtenues à partir de Mus musculus ơ, piégé le 11 mars 1967 à Novato Creek, Black Point ( ${ }^{\circ} 689$ M).

Souche 50-690 M (DJF 40). Matériel récolté à partir de lignées pures de Souris Mus musculus infestées expérimentalement (Expérience 67-8, 5 mai 1967). Cette souche a été obtenue à partir de Peromyscus maniculatus gambeli piégé près de Woodland par le $\mathrm{D}^{\mathrm{r}}$ Frank A. Ehrenford en 1950. Depuis ce temps elle a été maintenue chez la Souris de laboratoire aux U.S.A.

Souche 66-691 M (DJF 41). Matériel récolté à partir de Souris de lignée pure Mus musculus infestées expérimentalement (Expérience 66-8, 8 août 1968). Cette souche a été obtenue à partir d'une Souris sauvage Mus musculus piégée à Conway-Ranch en juin 1966.

Tube 694 (DJF 32). Matériel récolté à partir de Souris sauvage et de lignées pures (Mus musculus) et de quelques Reithrodontomys raviventris (Expérience 67-8, 5 mai 1967). Cette souche a été obtenue à partir du Reithrodontomys raviventris $\mathrm{n}^{\circ} 693 \mathrm{M}$. Les spécimens sont coparasites d'Heligmosomoides douglasi n. sp.

Tube 334 M (collection M. J. Kinsella). Souche de laboratoire chez Mus musculus. Matériel transmis par le $\mathrm{D}^{r}$ John Cross, San Francisco, Origine de la souche : Peromyscus maniculatus gambeli. (souche 50).

(1) Les spécimens des tubes $656 \mathrm{M}$ et $657 \mathrm{M}$ sont déposés dans les collections U.S.N.M. Helminthological Collection sous le $\mathrm{n}^{\circ} 72184$. 

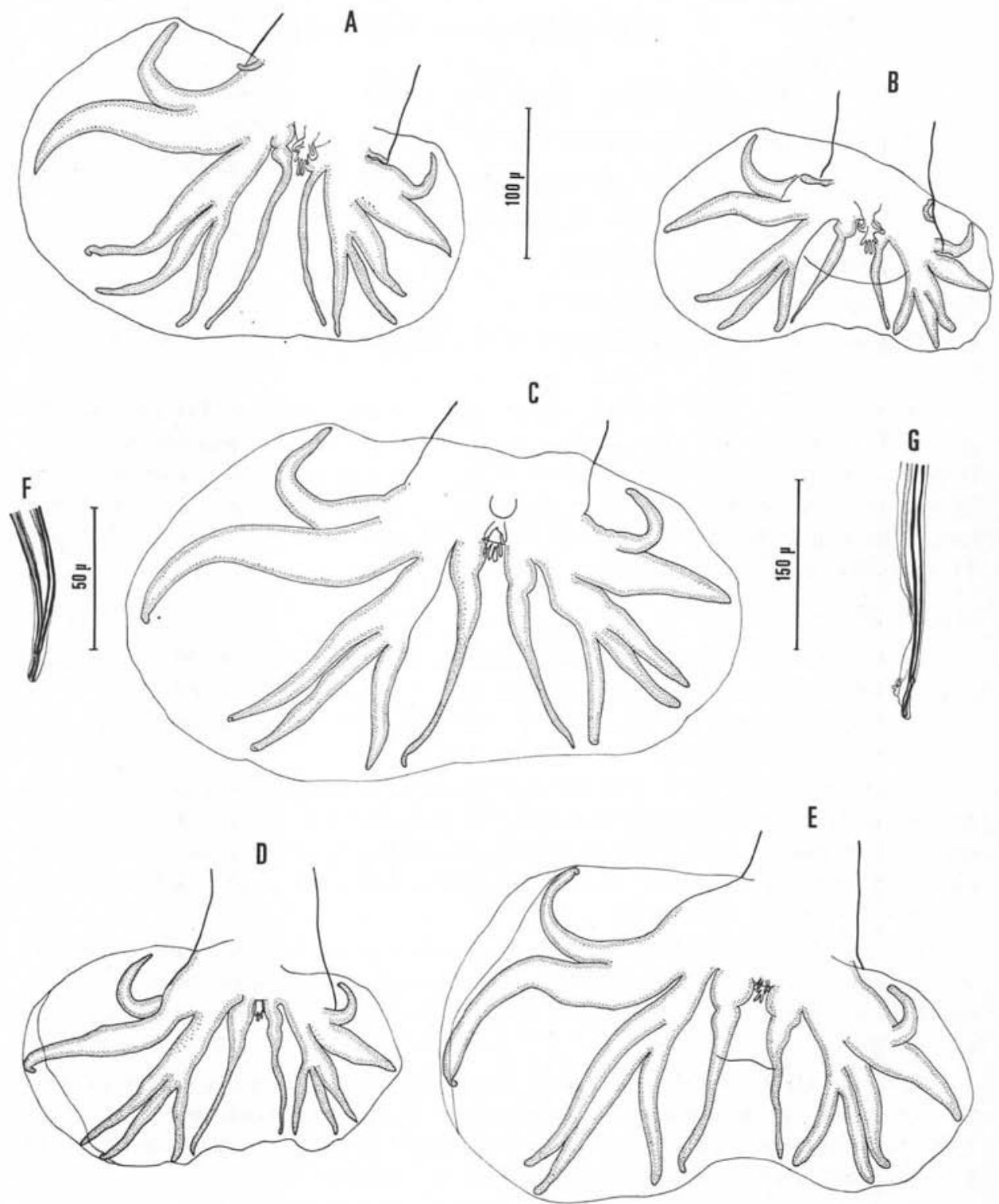

FIG. 1. - Bourses caudales. A, H. p. polygyrus (Dujardin, 1845) chez Apodemus sylvaticus de France. B, H. p. corsicus (Durette-Desset, 1968) chez Mus musculus brevirostris de Corse. C, H. p. bakeri n. sub. sp. chez Mus musculus d'Amérique du Nord. D, id chez Reithrodontomys megalotis d'Amérique du Nord. E, id chez Mus musculus d'Amérique du Nord (souche Ehrenford, 1950, supposée isolée de Peromyscus). Pointes de spicule. F, H. p. polygrus. G, H. p. bakeri, A, C, D, E, écả. : $150 \mu-$ B, éch. : $100 \mu-F, G$, éch. : $50 \mu$ 
2) Chez Reithrodontomys (Cricétidé).

a) 2 R. megalotis, tube 692 M (DJF 30), q piégées le 17 décembre 1966 à ConwayRanch, Woodland.

b) $8 R$. raviventris, tube $693 \mathrm{M}$ (DJF 31), ô piégés du 10 au 12 mars 1967 à Novato Creek, Black Point.

\section{Discussion.}

Les spécimens sont très proches d'Heligmosomoides p. polygyrus (Dujardin, 1845) et présentent en particulier la même disposition des côtes bursales avec un renflement basal des côtes externo-dorsales et des spicules toujours inférieurs à $600 \mu$ (420 à $520 \mu$ chez Mus musculus, $450 \mu$ chez Reithrodontomys megalotis, $490 \mu$ chez $R$. raviventris).

Cependant, certains éléments constants distinguent la forme néarctique : côte 4 gauche presqu'aussi longue que la côte 5 (fig. 1, C, D, E), alors que chez le polygyrus, elle est nettement plus courte (fig. 1, A) ; pointes des spicules toujours séparées (fig. 1, G) alors que chez le polygyrus elles sont enfermées dans une gaine (fig. 1, F); augmentation relative du nombre des crêtes cuticulaires par rapport à la longueur du corps (par exemple, pour une femelle de $15,5 \mathrm{~mm}$ de long, les crêtes cuticulaires, dans la partie postérieure du corps, sont au nombre de 35 chez le p. polygyrus et de 46 chez les spécimens américains (fig. 2).

Nous séparons donc les spécimens étudiés ci-dessus et proposons d'en faire une nouvelle sous-espèce: Heligmosomoides polygyrus bakeri $\mathrm{n}$. sub. $\mathrm{sp}$. en l'honneur du $D^{r}$ Norman F. Baker, School of Veterinary Medicine, University of California, Davis.

Remarques: Les spécimens d'Heligmosomoides polygyrus bakeri parasites des Souris (Mus musculus) sont particulièrement proches d'H. polygyrus corsicus (Durette-Derset, 1968 a) parasite de Mus musculus brevirostris du fait que le nombre de crêtes cuticulaires est très comparable (voir fig. 2). Ils s'en distinguent immédiatement cependant, du fait que chez des spécimens de même taille, la bourse caudale du bakeri est beaucoup plus grande (corsicus $230 \mu \times 120 \mu$; bakeri $540 \mu \times 270 \mu$ ) (fig. $1 \mathrm{~B}, \mathrm{C}, \mathrm{E}$ ).

Les spécimens provenant du Reithrodontomys ont une bourse caudale de taille intermédiaire $(350 \mu \times 180 \mu)$ et une côte 4 particulièrement longue (fig. 1, D). N'ayant à notre disposition que 3 spécimens, nous ne pouvons pas savoir si ces éléments sont suffisamment constants pour qu'il soit nécessaire de les séparer de bakeri.

\section{Heligmosomoides polygyrus americanus n. sub. sp.}

\section{Matériel étudié :}

1) Nombreux $\delta$ et $q$ (Matériel type) (tube $757 \mathrm{M}$ ), parasites d'un Phenacomys intermedius Merriam (Microtidé) récoltés par M. Chris Maser le 25 juillet 1969 dans l'Oregon (U.S.A.) et transmis par le $\mathrm{D}^{r}$ R. L. Rausch.

2) $1 \delta^{*}, 1$, provenant du même hôte et de la même région (tube $756 \mathrm{M}$ ). 


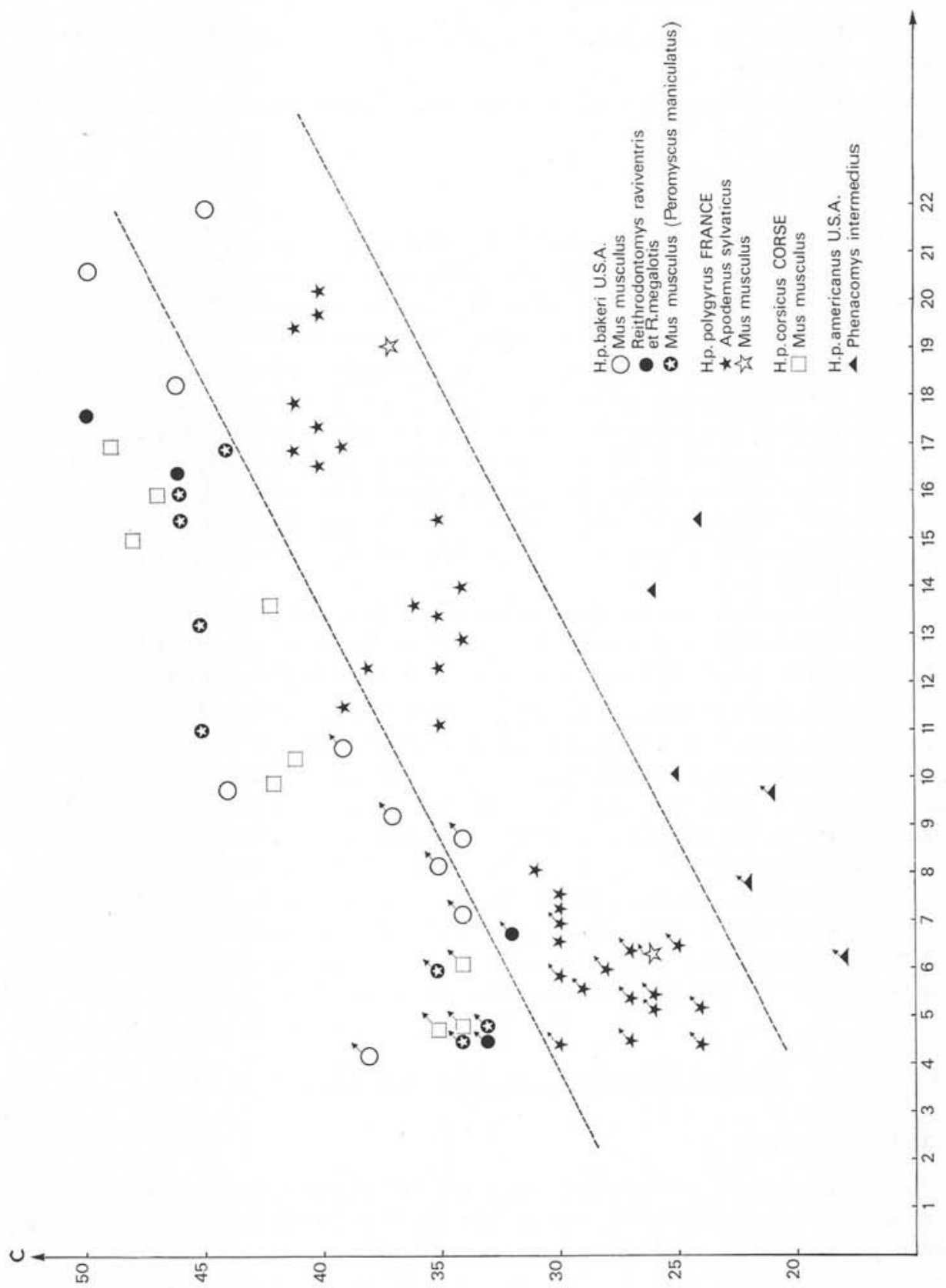


3) $3 \sigma^{\circ}, 5$, provenant du même hôte, récoltés le 24 avril 1965 dans le Montana (U.S.A.) (Collection J. M. Kinsella). Paratypes, U.S.N.M, Helminthological Collection No. 71976 .

\section{Diseussion.}

Ces parasites sont aussi très proches d'Heligmosomoides polygyrus polygyrus (Dujardin, 1845). Nous avons déjà noté (1968 a) qu'H. p. polygyrus est une espèce très polymorphe. Dans le matériel de France, nous avons trouvé un mâle que nous figurons (fig. 1, A) et qui présente, comme nos parasites un allongement important du tronc commun des côtes latérales droites.

Cependant, certains éléments constants distinguent la forme du Phenacomys. Ce sont l'allongement des spicules $(870-950 \mu$ au lieu de $500-750 \mu)$; la forme différente de l'extrémité des spicules (fig. 3, 4) et de la côte dorsale (fig. $3 \mathrm{D}$ ) ; l'absence de renflement basal des côtes externo-dorsales qui sont par contre pourvues d'une petite branche interne (fig. 3 F) ; la diminution du nombre des crêtes cuticulaires (par exemple, pour une femelle de $15,5 \mathrm{~mm}$ de long, les arêtes cuticulaires dans la partie postérieure du corps sont de $35 \mathrm{chez}$ p. polygyrus, de $24 \mathrm{chez}$ les spécimens des Phenacomys (fig. 2).

Nous séparons donc les spécimens des Phenacomys et en faisons une nouvelle sousespèce : Heligmosomoides polygyrus americanus n. sub. sp. (synonyme : Heligmosomum dubius sensu Kinsella, 1967).

Remarques : En 1954, Schad trouve $N$. dubius ( $=H$. polygyrus) chez des Rongeurs déterminés Microtus chrotorrhinus piégés à Seven Islands et à Fort McKenzie, Québec, Canada. Peterson (1962) examine à nouveau les Rongeurs de Schad et les identifie à Phenacomys ungava et non à $M$. chrotorrhinus. Nous avons examiné du matériel de Schad provenant de la collection de l'Institut de Parasitologie MacDonald College, Québec. Ce matériel comprenait une queue de mâle et deux femelles. Les spicules sont longs de $950 \mu$ il n'y a pas de renflement basal aux externo-dorsales et la morphologie de la pointe des spicules est celle de $H$. polygyrus americanus. Bien que n'ayant pu compter les crêtes cuticulaires à cause du nombre limité de spécimens, nous pensons qu'il est possible d'identifier le matériel de Schad à $H$. polygyrus americanus.

\section{Heligmosomoides douglasi $\quad$ n. sp.}

\section{Matériel étudié :}

1 के, 1 ? (types), parasites de 8 Reithrodontomys raviventris ơ piégés du 10 au 12 mars 1967 à Novato Creek, Black Point, Californie (collection D. J. Forrester). U.S.

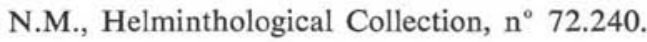

Fig. 2. - Varrations du nombre de crêtes cuticulaires (C) en fonction de la longueur du corps (L). Le diagramme fait apparaitre trois groupes: 1) $H$. p. americanus: $\mathrm{C}$ est faible par rapport à $\mathrm{L}$. 2) H. p. polygyrus : C est moyen par rapport à L. 3) H. p. bakeri et $H$. p. corsicus : C est élevé par rapport à L. Le signe "étoile blanche sur fond noir» représente les spécimens de Mus musculus infestés par la souche d'Ehrenford 1950 dont l'origine serait des parasites du Peromyscus maniculatus 
2 o 694 M (DJF 32), coparasites d'Heligmosomoides polygyrus bakeri. Matériel récolté à partir de Souris sauvages et de lignées pures (Mus musculus) et de quelques Reithrodontomys raviventris (Expérience 67-8, 5 mai 1967). Cette souche a été obtenue à partir de 8 Reithrodontomys raviventris $693 \mathrm{M}$ (collection D. J. Forrester).

\section{Description.}

Corps enroulé de façon senestre le long de la ligne ventrale selon plusieurs tours de spire, la partie postérieure du corps étant déroulée.

Chez le ơ, vésicule céphalique $120 \mu$. Deirides seitiformes. Axe des spicules dédoublé dans sa partie moyenne.

Synlophe: Dans sa partie moyenne, le corps est parcouru par des crêtes longitudinales au nombre de $32 \mathrm{chez}$ le $\delta^{\top}$ et de $41 \mathrm{chez}$ la ?. Les crêtes dorsales, ventrales et latérales droites naissent sur le bord postérieur de la vésicule céphalique (fig. 4, A, B, D), tandis que les crêtes latérales gauches naissent à différents niveaux sur le champ latéral (fig. 4, C) et donnent naissance en partie aux crêtes dorsales et ventrales. Les crêtes dis-

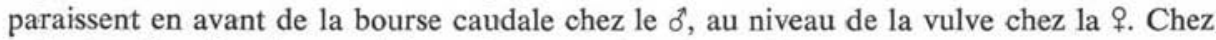
cette dernière, il existe aussi des crêtes entre la vulve et l'anus.

La pointe des crêtes est dirigée de la droite vers la gauche sur les 2 faces, l'orientation étant plus nette sur la face ventrale (fig. 4, E). Les crêtes dorsales sont un peu plus développées que les autres crêtes.

Mâle. Corps long de 5,9 mm, large de $100 \mu$ dans sa partie moyenne. Vésicule céphalique haute de $120 \mu \times 45 \mu$ de large. Anneau nerveux, pore excréteur et deirides situés respectivement à $230 \mu, 390 \mu$ et $395 \mu$ de l'apex. Esophage long de $570 \mu$.

Bourse caudale sub-symétrique (lobe droit : $200 \mu$ de large, lobe gauche : $180 \mu$ ) haute de $130 \mu$, lobe dorsal bien individualisé. Côtes prébursales présentes. Côtes 3 bien développées. Tronc commun aux côtes latérales très court. Côtes 8 relativement courtes. Côte dorsale divisée dans son $1 / 3$ postérieur en 4 rameaux de longueur sensiblement égale. Cône génital développé portant 2 longues papilles sur sa lèvre postérieure (fig. 4, H).

Spicules longs de $1 \mathrm{~mm}$, dédoublés dans leur partie moyenne, se terminant en une pointe longue de $15 \mu$ (fig. 4, I). Tout le lobe dorsal de la bourse caudale est occupé par une membrane (fig. $4, \mathrm{H}$ ).

Femelle. Corps long de $11,9 \mathrm{~mm}$, large de $150 \mu$ dans sa partie moyenne. Vésicule céphalique haute de $75 \mu \times 45 \mu$ de large. Anneau nerveux, pore excréteur et deirides situés respectivement à $160 \mu, 270 \mu$ et $280 \mu$ de l'apex. Esophage long de $560 \mu$.

Fig. 3. - Heligmosomoides polygyrus americanus n. sub. sp. A, $\delta$, détail des deirides, vue dorsale. $\mathrm{B}, \hat{\delta}$, coupe transversale au milieu du corps. C, + , ovejecteur disséqué. D, ô, cône génital et côte dorsale, vue ventraie. E, $\sigma^{\star}$ cône génital, vue latérale gauche. F, ơ , côtes dorsale et externo-dorsale, vue dorsale. $\mathrm{G}, \delta$, bourse caudale, vue ventrale. H, $\delta$, pointe d'un spicule. I, $q$, extrémité postérieure, vue latérale gauche. A, B, D, E, H, éch. : $50 \mu$. C, G, I, éch. : $150 \mu$. F, éch. : $100 \mu$ 

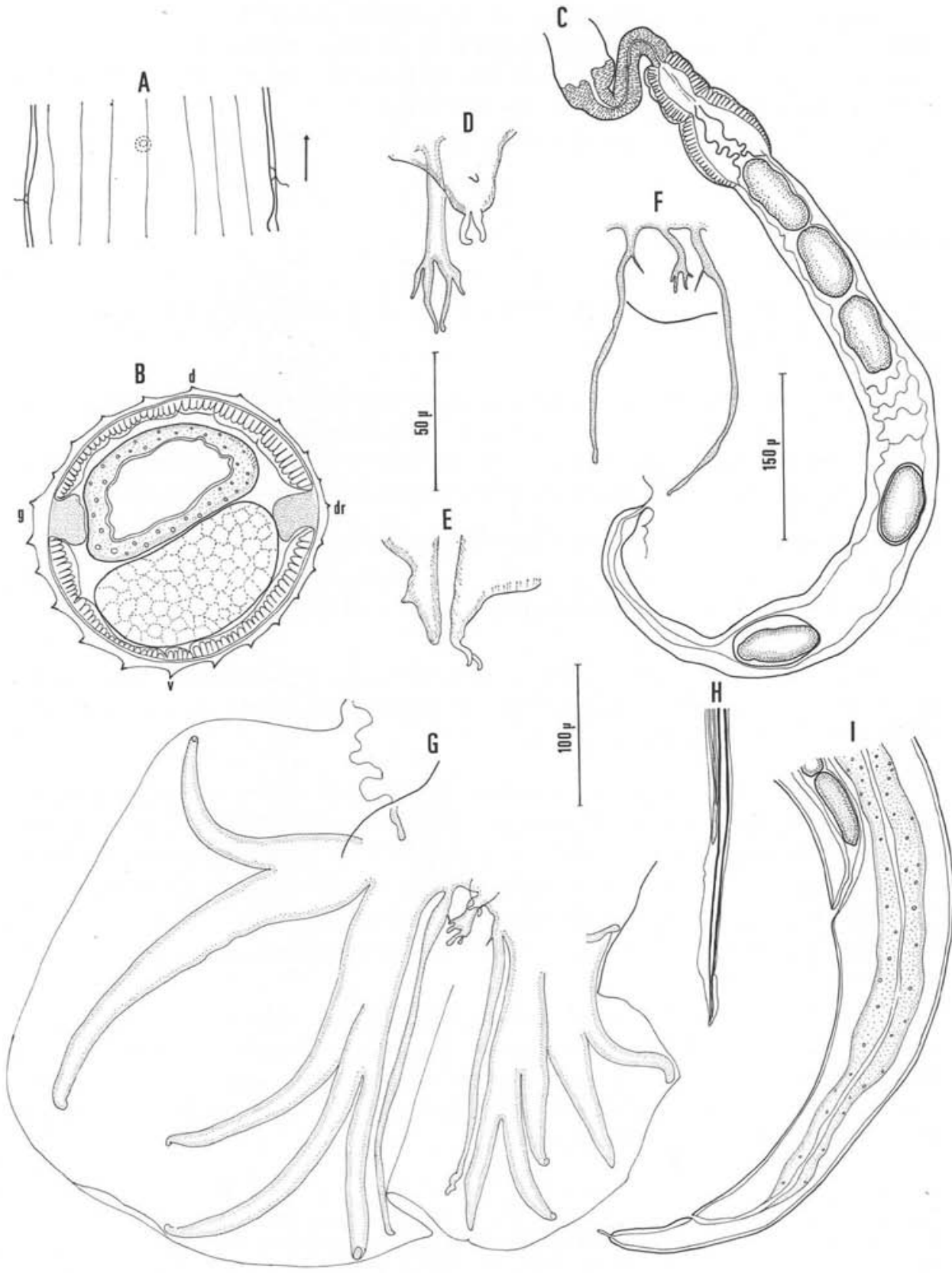
Appareil génital monodelphe avec un vestibule très allongé $(865 \mu)$; sa partie proximale s'élargit et est muscularisée. Sphincter long de $50 \mu$, trompe de $180 \mu$. L'utérus contient de très nombreux œufs au stade morula, longs de $60 \mu$ sur $40 \mu$ de large. La vulve s'ouvre à $268 \mu$ de la pointe caudale (fig. $4, \mathrm{G}$ ).

Queue longue de $130 \mu$, pourvue d'une pointe de $12 \mu$ (fig. 4, G).

\section{Discussion.}

Nos parasites présentent les caractères essentiels du genre Heligmosomoides Hall, 1916 sensu Durette-Desset, 1968.

Parmi les espèces néarctiques, nos spécimens sont proches d'Heligmosomoides bullosus bullosus Durette-Desset 1967, parasites de Microtus oeconomus en Alaska, avec une disposition semblable des côtes bursales, des externo-dorsales droites et non sinueuses, la présence d'une membrane bursale, un allongement comparable du vestibule.

Cependant nos spécimens se distinguent immédiatement par 2 caractères :

1) des spicules nettement plus courts (1 mm au lieu de $1,9 \mathrm{~mm}), 2$ ) des crêtes cuticulaires en nombre beaucoup plus élevé ( $30 \mathrm{chez}$ le $\delta$ au lieu de 17, $41 \mathrm{chez}$ la $q$ au lieu de 21).

Nous séparons donc notre matériel et nous proposons de le nommer Heligmosomoides douglasi $\mathrm{n}$. sp. en l'honneur du $\mathrm{D}^{\mathrm{r}}$ James R. Douglas, School of Veterinary Medicine, University of California, Davis.

Remarques: $H$. douglasi est un parasite de Cricétidé, mais il doit cependant être rattaché aux Heligmosomoides parasites de Microtidés néarctiques et non au groupe polygyrus. En effet, la bourse caudale est du type bullosus, les spicules mesurent $1 \mathrm{~mm}$ et le nombre de crêtes cuticulaires est pratiquement constant tout au long du corps.

Il est intéressant de noter que l'espèce dont il se rapproche le plus $H$. bullosus bullosus paraît une des espèces les moins évoluées des Heligmosomoides néarctiques (cf. Durette-Desset, 1968 b).

Nous interprétons $H$. douglasi comme un parasite de capture à partir des parasites de Microtidés. Cette spéciation, comme chez les espèces holarctiques connues chez des hôtes de capture, entraîne une augmentation du nombre des crêtes cuticulaires. Par contre, les spicules sont relativement courts par rapport à ceux des Heligmosomoides parasites de Microtidés néarctiques.

Fig. 4. - Heligmosomoides douglasi n. sp. A, B, C, D, $\widetilde{o}$, naissance des crêtes cuticulaires, successivement, vue dorsale, vue ventrale, vue latérale gauche, vue latérale droite. $\mathrm{E}, \delta$, coupe transversale au milieu du corps. F, $\delta^{\star}$, extrémité antérieure, vue latérale gauche. G, $q$, extrémité postérieure, vue ventrale. H, $\delta$, bourse caudale, vue ventrale. I, ơ, pointe d'un spicule. A, B, C, D, éch. : $100 \mu$. E, I, éch. : $50 \mu . \mathrm{G}, \mathrm{H}$, éch. : $150 \mu$ 


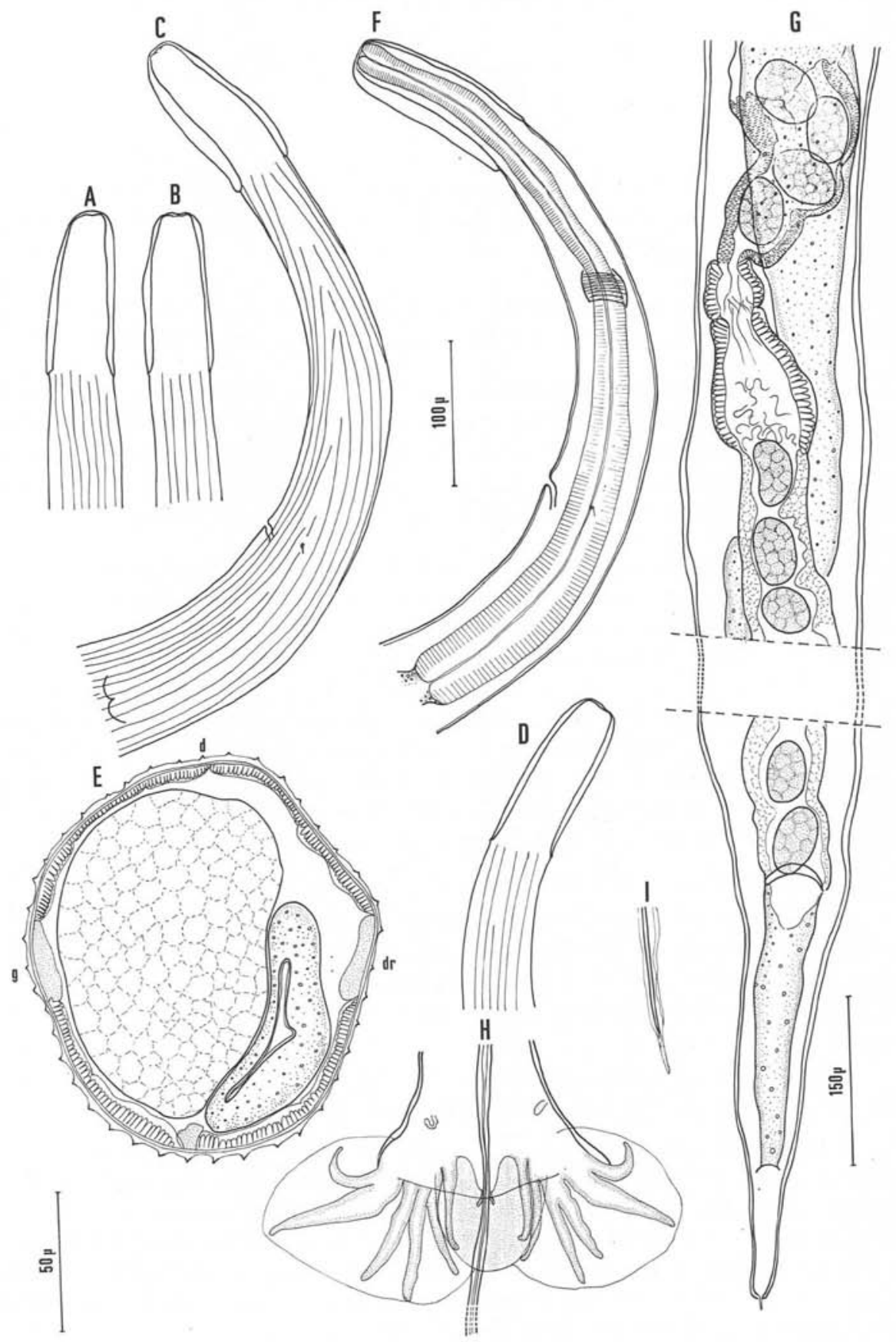




\section{CONOLUSION}

Comme l'un de nous a tenté de le démontrer dans un précédent travail (DuretteDesset 1967), l'origine des Nématodes Heligmosomoides parasites de Rongeurs néarctiques paraît être l'Europe centrale.

Cependant l'introduction de ces Vers en Amérique du Nord s'est faite par deux voies différentes (fig. 5), ce qui permet de les séparer en 2 groupes distincts (Tableau 1) :

1) le premier groupe est essentiellement inféodé aux Microtidés. Dans notre conception, les spéciations ont eu lieu progressivement au fur et à mesure que les populations de Microtidés s'éloignaient de leur lieu d'origine : l'Europe centrale. L'introduction de ce groupe en Amérique du Nord a eu lieu au Pleistocène en même temps que le passage des Microtidés d'Europe en Amérique par le détroit de Bering;

2) le deuxième groupe, constitué actuellement par deux sous-espèces du polygyrus européen, s'est introduit en Amérique du Nord en même temps que la Souris Mus musculus. Cette introduction est contemporaine de l'homme et a dû s'effectuer par passage sur bateaux à travers l'Atlantique.

En France, Heligmosomoides p. polygyrus passe sans modifications du Mulot (Apodemus sylvaticus) à la Souris (Mus musculus). Par contre, il subit une sub-spéciation en Corse lorsqu'il s'adapte à Mus musculus brevirostris pour donner H. p. corsicus. Le même phénomène se produit en Amérique du Nord où la forme de Mus musculus H. p. bakeri est une sous-espèce de l'espèce type européenne.

Cette sous-espèce se rencontre également chez un Cricétidé : Reithrodontomys.

Le passage au Phenacomys intermedius (Microtidé) se traduit par une nouvelle subspéciation qui entraîne un allongement des spicules (phénomène habituel dans l'évolution du genre Heligmosomoides) et aussi une diminution du nombre des crêtes cuticulaires alors que l'évolution du genre implique normalement un accroissement de ce nombre.

Cette seconde spéciation chez Phenacomys s'explique aisément du fait que ce Rongeur vit en populations très isolées (fide R.L. Rausch).

Nous n'avons aucune preuve expérimentale pour donner le statut d'espèce ou de sous-espèce aux différentes formes étudiées ci-dessus. Nous avons attribué un niveau d'espèce aux spéciations successives qui s'étagent entre l'Europe centrale et l'Amérique du Nord par le détroit de Bering, car il n'y a pas eu de barrière géographique précise et le brassage ultérieur des populations est vraisemblable. Dans le cas des formes rattachables à polygyrus, au contraire, il s'agit d'un isolement brutal et il est très possible que des croisements expérimentaux soient parfaitement féconds. 


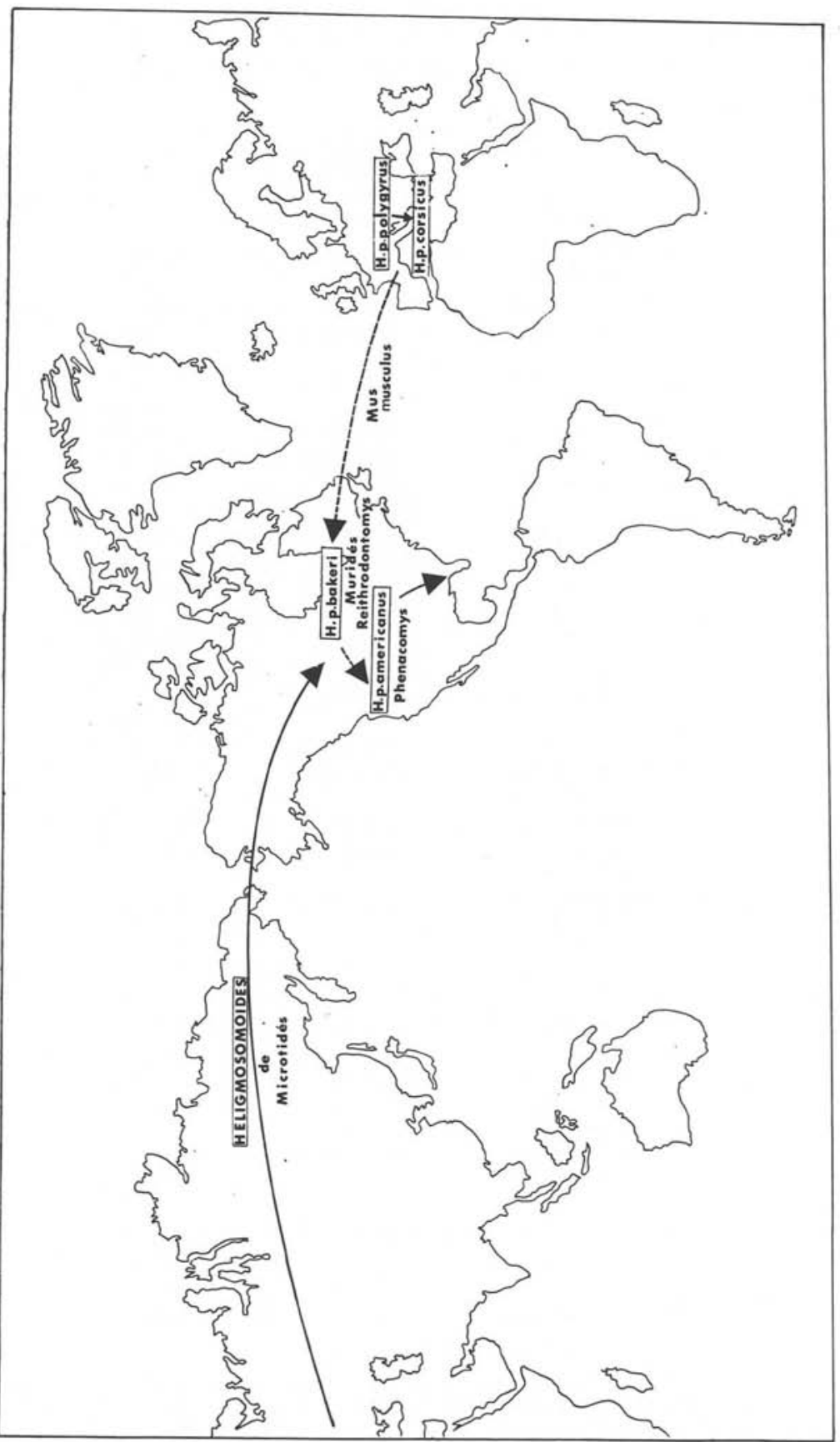

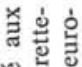
号 . ฏ. 엄코 离造导 है

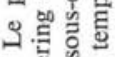
में 웜 는 氙运 ญั 营导出

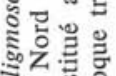
ปี่ \&ै 을 语合的

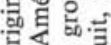
등명 ผ 岂氙 훌현 空密 1 : เ 它它岇 
Tableau I. - LISTE des espèces D' Heligmosomoides DE RONGEURS D'AMÉRIQUE DU NoRD

\begin{tabular}{|c|c|c|c|}
\hline Espèce & Hôte & Région & Référence \\
\hline \multicolumn{4}{|l|}{ Groupe 1: } \\
\hline H. carolinensis $\ldots$. & Clethrionomys gapperi & $\begin{array}{l}\text { North Carolina } \\
\text { Maryland } \\
\text { Québec }\end{array}$ & $\begin{array}{l}\text { Dikmans, } 1940 \\
\text { Lichtenfels } \\
\text { et Haley, } 1968 \\
\text { Schad, } 1954\end{array}$ \\
\hline H. longispiculatus .. & $\begin{array}{l}\text { Microtus pennsylvanicus } \\
\text { Microtus longicaudus } \\
\text { Ondatra zibethica }\end{array}$ & $\begin{array}{l}\text { Washington, D.C. } \\
\text { Alaska } \\
\text { New Jersey }\end{array}$ & $\begin{array}{l}\text { Dikmans, } 1940 \\
\text { Rausch, } 1952 \\
\text { Dikmans, } 1940\end{array}$ \\
\hline H. microti $\ldots \ldots \ldots$ & Microtus montanus & Wyoming & $\begin{array}{l}\text { Kuns } \\
\text { et Raush, } 1950\end{array}$ \\
\hline & $\begin{array}{l}\text { Microtus richardsoni } \\
\text { Microtus longicaudus } \\
\text { Microtus pennsylvanicus }\end{array}$ & $\begin{array}{l}\text { Wyoming } \\
\text { Montana } \\
\text { Montana }\end{array}$ & $\begin{array}{l}\text { Kuns } \\
\text { et Raush, } 1950 \\
\text { Kinsella, } 1967 \\
\text { Kinsella, } 1967\end{array}$ \\
\hline \multirow[t]{2}{*}{ H. bullosus bullosus } & $\begin{array}{l}\text { Microtus oeconomus } \\
\text { innuitus }\end{array}$ & Alaska & $\begin{array}{l}\text { Durette-Desset, } \\
1968 b\end{array}$ \\
\hline & M. o. operarius & Alaska & $\begin{array}{l}\text { Durette-Desset, } \\
1968 b\end{array}$ \\
\hline $\begin{array}{l}\text { H. bullosus } \\
\text { matthewensis .... }\end{array}$ & $\begin{array}{l}\text { Microtus abbreviatus } \\
\text { fischeri }\end{array}$ & Alaska & $\begin{array}{l}\text { Durette-Desset, } \\
1968 b\end{array}$ \\
\hline H. tenorai...... & $\begin{array}{l}\text { Lemmus sibiricus } \\
\quad \text { alascensis }\end{array}$ & Alaska & $\begin{array}{l}\text { Durette-Desset, } \\
1968 b\end{array}$ \\
\hline H. montanus ...... & Microtus longicaudus & Alaska & $\begin{array}{l}\text { Durette-Desset, } \\
1968 b\end{array}$ \\
\hline H. wisconsinensis .. & $\begin{array}{l}\text { Microtus californicus } \\
\text { Microtus pennsylvanicus } \\
\text { pennsylvanicus }\end{array}$ & $\begin{array}{l}\text { California } \\
\text { Wisconsin }\end{array}$ & $\begin{array}{l}\text { Ce travail } \\
\text { Durette-Desset, } \\
1968 b\end{array}$ \\
\hline H. hudsoni ... & $\begin{array}{l}\text { Dicrostonyx hudsonius } \\
\text { Dicrostonyx torquatus }\end{array}$ & $\begin{array}{l}\text { Canada } \\
\text { Alaska }\end{array}$ & $\begin{array}{l}\text { Cameron, } 1937 \\
\text { Durette-Desset, } \\
1968 b\end{array}$ \\
\hline H. douglasi...$\ldots$. & $\begin{array}{l}\text { Reithrodontomys } \\
\text { raviventris }\end{array}$ & California & ce travail \\
\hline \multirow{3}{*}{$\begin{array}{l}\text { Groupe } 2: \\
H . \text { polygyrus } \\
\quad \text { bakeri (1) } \ldots \ldots\end{array}$} & & & \\
\hline & Mus musculus & California & Forrester, 1971 \\
\hline & $\begin{array}{l}\text { Peromyscus } \\
\text { maniculatus } \\
\text { Reithrodontomys } \\
\text { megalotis } \\
\text { Reithrodontomys } \\
\text { raviventris }\end{array}$ & $\begin{array}{l}\text { California } \\
\text { California } \\
\text { California } \\
\text { California } \\
\text { California }\end{array}$ & $\begin{array}{l}\text { Ehrenford, } 1954 \\
\text { ce travail } \\
\text { Forrester, } 1971 \\
\text { ce travail } \\
\text { Forrester, } 1971 \\
\text { ce travail }\end{array}$ \\
\hline \multirow[t]{2}{*}{$\begin{array}{l}H . \text { polygyrus } \\
\quad \text { americanus } \ldots . . .\end{array}$} & Phenacomys intermedius & $\begin{array}{l}\text { Montana } \\
\text { Idaho } \\
\text { Orégon }\end{array}$ & $\begin{array}{l}\text { Kinsella, } 1967 \\
\text { Kinsella, } 1967 \\
\text { ce travail }\end{array}$ \\
\hline & Phenacomys ungava & Québec & Schad, 1954 \\
\hline
\end{tabular}

(1) D'autres auteurs ont décrit sous le nom de «Nematospiroides dubuus . des Nématodes qui correspondaient probablement à $H$. polygyrus bakeri: Babero et Mathias, 1967 chez Peromyscus maniculatus et Peromyscus eremicus; Hall, Sonnenberg et Hodes, 1955 chez Mus musculus et Peromyscus leucopus. 


\section{Bibliographie}

Babero (B. B.) and Matthias (D.), 1967. - Protospirura peromysci n. sp. (Nematoda : Spiruridae) and other helminths from Peromyscus spp. in Nevada. Proc. Helm. Soc. Wash., 34, 255-261.

BAKER (N. F.), 1954. - Trichostrongylidosis - the mouse as an experimental animal. Proc. Book, Am. Vet. Med. Assoc., 91, 185-191.

Cameron (T. W. M.), 1937. - A new species of Heligmosominae from the Labrador collaredlemming. Papers on Helminthology pub. in commemoration of the 30 year jubilium of K. I. Skrjabin and of 15th anniv. of the All-Union Institute of Helminthology, Moscow, pp. 66-68. In Skrjabin et al., 1954.

Cross (J. H.), 1960. - The natural resistance of the white rat to Nematospiroides dubius and the effect of cortisone on this resistance. J. Parasit., 46, 175-185.

Dikmans (G.), 1940. - A note on the genera Nematospiroides Baylis, 1926 and Sincosta Roe, 1929 (Nematoda, Heligmosomidae), with descriptions of two new species of Nematospiroides. Proc. Helm. Soc. Wash., 7, 79-82.

Douglas (J. R.), BaKer (N. F.) and Longhurst (W. M.), 1956. - The relationship between particle size and anthelmintic efficiency of phenothiazine. Am. J. Vet. Res., 17, 318-323.

Durette-Desset (M.-Cl.), 1967. - Evolution des Nématodes Héligmosomes en rapport avec celle de leurs hôtes fondamentaux, les Microtidae. C.R. Acad. Sc. Paris, 265, 15001503 .

—, $1968 a$. - Identification des Strongles des Mulots et Campagnols décrits par Dujardin. An. Parasitol., 43, 387-404.

—, 1968 b. - Les systèmes des crêtes cuticulaires chez les Nématodes Héligmosomes. III. Etude de sept espèces parasites de Rongeurs néarctiques et rétablissement du genre Heligmosomoides Hall, 1916. Bull. Mus. Nat. Hist. Nat., 40, 186-209.

EHRENFord (F. A.), 1954. - The life cycle of Nematospiroides dubius Baylis (Nematoda: Heligmosomidae). J. Parasit., 40, 480-481.

ForRester (D. J.), 1971. - Heligmosomoides polygyrus (= Nematospiroides dubius) from wild rodents of northern California : natural infections, host specificity, and strain characteristics. J. Parasit., 57, 498-503.

Hall (J. E.), Sonnenberg (B.) and Hodes (J. R.), 1955. - Some helminth parasites of rodents from localities in Maryland and Kentucky. J. Parasit., 41, 640-641.

Lichtenfels (J. R.) and HALEY (A. J.), 1969. - New host records of intestinal nematodes of Maryland rodents and suppression of Capillaria bonnevillei Grundmann and Frandsen, 1960 as a synonym of C. americana Read, 1949. Proc. Helm. Soc. Wash., 35, 206-211.

Kinsella (J. M.), 1967. - Helminths of Microtinae in western Montana. Can. J. Zool., 45, 269-274.

KUNS (M. L.) and RAUSCH (R. L.), 1950. - An ecological study of helminths of some Wyoming voles (Microtus spp.) with a description of a new species of Nematospiroides (Heligmosomidae: Nematoda). Zoologica, N.Y. Zool. Soc., 35, 181-188. 
Peterson (R. L.), 1962. - Notes on the distribution of Microtus chrotorrhinus. J. Mammal., 43,420 .

RAUSCH (R. L.), 1952. - Studies on the helminth fauna of Alaska. XI. Helminth parasites of Microtine rodents, taxonomic considerations. J. Parasit., 38, 415-444.

Ró́ (G.C.), 1929. - A new nematode, Sincosta aberrans, new genus, and new species from a rodent. Proc. U.S. Nat. Mus., 75, 1-3.

SCHAD (G. A.), 1954. - Helminth parasites of mice in north eastern Quebec and the coast of Labrador. Can. J. Zool., 32, 215-224.

Skrjabin (K. I.), Shikhobalova (N. P.) et Schulz (R. S.), 1954. - Principles of nematodology ed. by K. I. Skrjabin. Vol. IV. Dictyocaulidae, Heligmosomatidae, and Ollulanidae of animals. Moscow: Izd. Akad. Nauk S.S.S.R., 319 pp. (In Russian).

SPURLOCK (G. M.), 1943. - Observations on host-parasite relations between laboratory mice and Nematospiroides dubius Baylis, J. Parasit., 29, 303-311. 